Project ID number: EMSP 55031

\title{
Genetic Analysis of Stress Responses in Soil Bacteria for Enhanced Bioremediation of Mixed Contaminants
}

\section{Principal Investigator:}

Kwong-Kwok Wong, Ph.D.

Pacific Northwest National Laboratory

P.O. Box 999, MSIN P7-56

Richland, WA 99352

(509) 375-5097 (phone)

kk.wong@pnl.gov

\section{Research Objective:}

The purpose of this project is to provide fundamental knowledge on environment stress response of subsurface bacteria and a radiation-resistant bacterium (Deinococcus radiodurans). This information will be particularly useful in the development of successful bioremediation strategies. These organisms represent two phylogenetically distinct groups of soil bacteria, each of which has specific features of interest for bioremediation. The subsurface bacteria, Sphingomonas spp (Savannah River Site), have unique degradative capabilities of organic waste. The study of subsurface bacteria will serve as a model for bioremediation using indigenous bacteria. $D$. radiodurans exhibits high resistance to external stress such as ionizing radiation and organic solvent. The study of $D$. radiodurans will serve as a model for the use of genetically engineered bacteria for bioremediation.

\section{Research Progress and Implications :}

This report summarizes the work that has been accomplished after the completion of a 3-year project, which started in October 1996. In our past 3-year studies, we have chosen two different bacteria as models for the understanding their genetic responses to starvation and solvent. One of the model organisms, Deinococcus radiodurans, is a stress-resistant bacterium. We have identified several genes that response to organic solvent and starvation, as well as several genes that are required for oxidative or radiation resistance (Markillie et al., 1999). The other model organism is Sphingomonas aromaticivorans, which is a subsurface bacterium with a $184 \mathrm{~kb}$ catabolic plasmid, and is able to degrade many organopollutants. We have completely sequenced the $184 \mathrm{~kb}$ catabolic plasmid (Wong et al., 1996; Romine et al., 1999), and were able to identify many genes involved in the degradation of organopollutants as well as genes related to the conjugative transfer of this plasmid to other bacteria. The solvent inducible genes will be useful for enhancing the solvent resistance of pollutant degrader such as Sphingomonas aromaticivorans strains, while the availability of the catabolic plasmid pNL1 will be useful for enhancing the pollutant degradative capabilities of $D$. radiodurans.

To identify solvent-inducible genes, total RNA was extracted from $D$. radiodurans that have been treated with xylene, trichloroethylene, or toluene. An RNA fingerprinting technique (Wong and McClelland, 1994) was used to identify the RNA transcripts that were induced by solvent. Four putative solvent-inducible genes were identified and were cloned. Two of these genes were found to be the rhamnosyl transferase and methyl transferase by sequencing analysis; the other two genes were unknown. Since the sensitivity of bacteria to solvent is related to their cell surface hydrophobicities, it is possible that these induced genes may be involved in changing the cell surface hydrophobicities. However, further studies are needed to prove that hypothesis. 
Furthermore, we have also shown that a member associated protein $p s p A$ is required for solvent resistance.

Using similar strategy, two starvation inducible genes that share sequence homology with $g l g X$, glycogen debranching enzyme and $h b d$, 3-hydroxybutyryl coenzyme A dehydrogenase. The enzymes are involved in the utilization of glycogen and fatty acid stored within the cell. The results indicated that $D$. radiodurans might utilize reserved glycogen and fatty acid during starvation condition. This may also explain the difference in fatty acid profile between starved bacteria and normal bacteria.

Enhanced in situ removal of hazardous wastes by stimulating growth of indigenous bacteria with nutrients is a current strategy for enhanced bioremediation. To test the effect of nutrient supply on subsurface bacteria, four subsurface bacteria, Terrabacter sp. B511, Arthrobacter sp. B518, Sphingomonas aromaticivorans B522, and Acinetobacter sp. B525, were chosen for the study. Strain B522 is an organic degrader; the other strains are not. We found that when high level of nutrient is added to starved bacteria, they response similarly in growth rate. However, when limiting amount of nutrient is added, B518 is stimulated to multiply with the highest growth rate. Our result indicates that the pollutant degrader may be eliminated if low nutrient supply is used repeatedly when there is a fast-growing bacterial strain in the microbial communities, such as the B518 in our case. The result suggest that stimulating indigenous bacteria for enhancing bioremediation may not be effective but harmful to the environment by depicting useful bacteria. However, further in situ experiments are required to support this hypothesis.

\section{Planned Activities:}

A proposal was submitted for the following planned activities. 1) we propose to use the fundamental knowledge that we have learnt to enhance the capabilities of Deinococcus radiodurans and Sphingomonas strains for bioremediation. 2) With the complete DNA sequences of pNL1 that we have determined, we propose to study the effect of stresses on the genetic transfer of such catabolic plasmid to other bacteria. 3) Finally, we would like to further investigate how nutrient supply will affect the relative growth of starved subsurface organic pollutant degraders in a microbial community.

\section{Publications :}

Romine, M., L.C. Stillwell, K.K. Wong, S. J. Thurston, E.C. Sisk, J. D. Saffer, and J. K. Fredrickson. 1999. Complete sequence of a 184.5-kb catabolism plasmid from Sphingomonas aromaticivorans strain F199. Journal of Bacteriology 181:1585-1602.

Markillie, L.M., S. Varnum , P. Hradecky, and K.K. Wong. 1999. Targeted mutagenesis by duplication insertion in the radioresistant bacterium Deinococcus radiodrans: radiation sensitivities of catalase $(\mathrm{katA})$ and superoxide dismutase $(\operatorname{sod} A)$ mutants. Journal of Bacteriology 181:666-669.

Wong, K.K., L. C. Stillwell, C. A. Dockery, and J.D. Saffer. 1996. Use of tagged random hexamer amplification (THRA) to clone and sequence minute quantities of DNA application to a $180 \mathrm{~kb}$ plasmid isolated from Sphingomonas F199. Nucleic Acids Res. 24: 3778-3783. 